\title{
INTRAOCULAR PRESSURE ESTIMATION USING PROPER ORTHOGONAL DECOMPOSITION
}

\author{
N. Botha ${ }^{1,2}$, S. Kok ${ }^{1}$, H. M. Inglis ${ }^{2}$
}

${ }^{1}$ Advanced Mathematical Modelling, Modelling and Digital Sciences, Council for Scientific and Industrial Research, South Africa (nbotha1@csir.co.za)

${ }^{2}$ Department of Mechanical and Aeronautical Engineering, University of Pretoria, South Africa

\begin{abstract}
Glaucoma is the second leading cause of irreversible blindness. The primary indicator for glaucoma is an elevated intraocular pressure, which is estimated by means of contact or non-contact tonometry. However, these techniques do not accurately account for the cornea properties that deviate from the norm, thus leading to the inaccurate estimation of the intraocular pressure. This work builds on a previous study, in which a combination of an artificial neural network and a genetic algorithm was used to estimate the intraocular pressure and cornea properties. This paper proposes to use proper orthogonal decomposition to accurately estimate the intraocular pressure independent of the cornea properties. The results indicate that proper orthogonal decomposition is able to estimate the intraocular pressure, and that the cornea properties have a slight influence on the estimation. For thicker corneas, however, the intraocular pressure prediction is influenced. This study concluded that this deterministic technique avoids the ambiguity that could result from a method relying on a stochastic optimization routine.
\end{abstract}

Keywords: intraocular pressure, glaucoma, cornea, proper orthogonal decomposition, anisotropic material.

\section{INTRODUCTION}

It is estimated that by 2020, 79.6 million people world wide will have glaucoma, an eye disease that permanently damages the optic nerve [15]. This makes it the second leading cause of blindness, after cataracts, and the primary cause of irreversible blindness.

As the intraocular pressure (IOP) is the primary indicator for glaucoma, it needs to be determined with accuracy. A popular method of estimating intraocular pressure is Goldmann applanation tonometry (GAT), which measures the indentation resistance of the cornea. Unfortunately this contact tonometry technique is not without its inaccuracies. Due to the calibration of the instrument, using the average populations' cornea properties, the intraocular pressure is estimated inaccurately for patients whose cornea properties deviate from the norm. Several studies have shown that the intraocular pressure determined from contact tonometry 
(Goldmann applanation) is not only dependent on the central corneal thickness (CCT) but on the corneal material properties as well $[2,7,9,12]$.

Ghaboussi et al. [6] suggested a non-invasive technique using a combination of a genetic algorithm (GA) and an artificial neural network (ANN) to estimate the intraocular pressure accurately. The proposed method assumes that a Goldmann applanation tonometer can be modified to measure the entire optical response history during applanation, rather than obtaining a single measurement at the optimum point of applanation. An axisymmetric finite element model of the cornea was developed using a transverse isotropic Fung material model to numerically simulate applanation tonometry. The intraocular pressure is then estimated by comparing the force-displacement response from the numerical simulation with the target response. There are two shortcomings with this approach. Firstly, the material model used for their finite element model of the cornea does not accurately represent the complex internal structure of the cornea, and secondly the combination of a genetic algorithm and an artificial neural network could lead to non-deterministic results.

This paper proposes to use a reduced order modelling technique known as proper orthogonal decomposition (POD), instead of the genetic algorithm and artificial neural network, to accurately estimate the intraocular pressure. A three dimensional orthotropic composite fiber material model is also implemented to capture the complex corneal behaviour more accurately.

\section{MATERIALS AND METHODS}

\subsection{Finite element model of the cornea}

The simplest mathematical model used to describe the aspherical shape of the cornea is a rotationally symmetric conicoid [1]:

$$
\left(x-x_{0}\right)^{2}+\left(y-y_{0}\right)^{2}+(1+Q)\left(z-z_{0}\right)^{2}-2 \mathrm{R}\left(z-z_{0}\right)^{2}=0,
$$

where $\mathrm{R}$ is the radius of curvature at the corneal apex, $Q$ is the surface asphericity parameter, $x$ is the distance along the equator axis, $y$ is the distance along the sagittal axis and the optical axis ( $z$ - axis) is the axis of rotation. Table 1 lists the average cornea dimensions, along with suggested values for the radius of curvature and surface asphericity parameter $[9,11,16]$.

Table 1. Average suggested values for cornea dimensions.

\begin{tabular}{ccc}
\hline & \multicolumn{2}{c}{ Average values } \\
\cline { 2 - 3 } & Anterior & Posterior \\
\hline Radius of curvature & $7.77 \mathrm{~mm}$ & $6.4 \mathrm{~mm}$ \\
Asphericity parameter & -0.18 & -0.60 \\
Diameter & $11.7 \mathrm{~mm}$ & - \\
\hline
\end{tabular}

The boundary conditions for the finite element model were applied as shown in Figure 1. For the inflation test, Figure 1(a), the boundary at the limbus is completely fixed to simulate the experimental conditions in Elsheikh et al. [5]. To simulate Goldmann applanation 
tonometry, as in Figure 1(b), the boundary at the limbus was fixed to only allow translation at an angle of $23^{\circ}$. Elsheikh et al. [4] found this to be the optimum representation of the actual connection of the cornea to the sclera. As the cornea is symmetric about the equator (xz) and sagittal (yz) planes, only a quarter model of the GAT simulation will be used, with symmetry boundary conditions. The intraocular pressure due to the aqueous humor flow in the anterior chamber is simulated with an uniform pressure distribution normal to the posterior surface of the cornea.

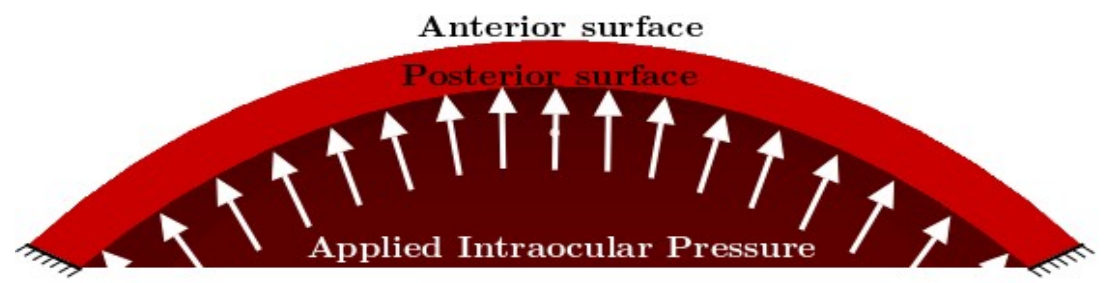

(a) Inflation experiment

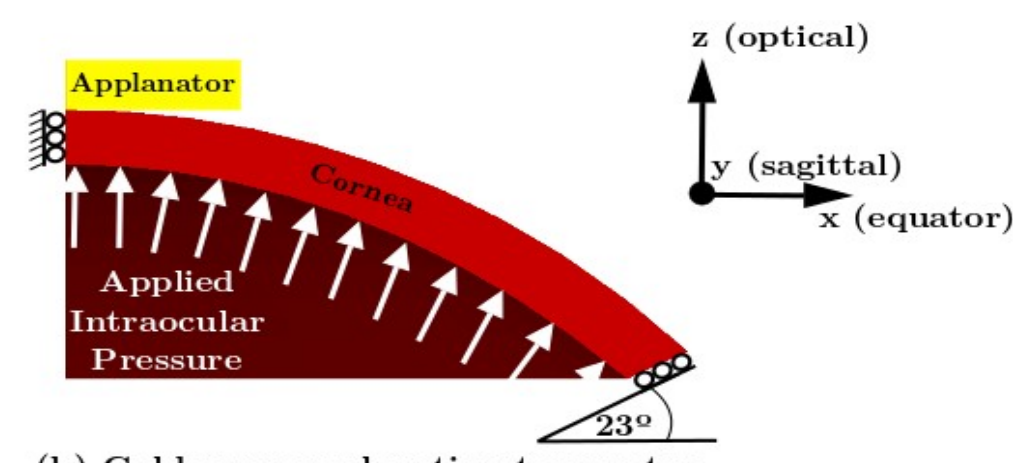

(b) Goldmann applanation tonometry

Figure 1: Front section view of the cornea model describing the boundary conditions for (a) the inflation experiment and (b) Goldmann applanation tonometry.

To represent the complex structure of the cornea the constitutive model chosen for this study is an anisotropic model with an isotropic neo-Hookean base and an exponential term to describe the response of embedded fibres [3]. The strain-energy density function is described mathematically [3]:

$$
U=C_{10}\left(\bar{I}_{1}-3\right)+\frac{1}{D_{1}}(J-1)^{2}+\sum_{i=1}^{n} \frac{k_{1 \mathrm{i}}}{2 \mathrm{k}_{2 \mathrm{i}}}\left[e^{\left\langle\left\langle\bar{I}_{4 \mathrm{i}}-1\right\rangle^{2}\right.}-1\right],
$$

where $C_{10}$ and $k_{1 \mathrm{i}}$ are stress-like material parameters (units of $\mathrm{MPa}$ ), $D_{1}$ is an incompressibility constant with a value of 0.004 and $k_{2 \mathrm{i}}$ is a dimensionless parameter. This model was chosen as it has been demonstrated to adequately describe biomaterials [14] and is already available in Calculix [3], an open-source finite element solver.

\subsection{Calibration of material coefficients from inflation test data}

Corneal biomechanical properties are a crucial element to not only understand the 
corneal behaviour and responses, but to accurately quantify other properties such as intraocular pressure. The inflation test is most popularly utilized to obtain these properties. A whole cornea specimen, which is clamped onto a pressure chamber, is inflated beyond its physical limitations. The material coefficients required to describe the cornea behaviour is found by calibration of a numerical model of the inflation test with experimental data [5]. A finite element model of the inflation test was constructed using the geometrical data as given in Table 2 [5].

Table 2. Cornea geometrical data used for the inflation test [5].

\begin{tabular}{cc}
\hline & Values \\
\hline Anterior radius of curvature & $7.5 \mathrm{~mm}$ \\
Posterior radius of curvature & $6.49 \mathrm{~mm}$ \\
Anterior Diameter & $11.252 \mathrm{~mm}$ \\
Central corneal thickness & $0.572 \mathrm{~mm}$ \\
\hline
\end{tabular}

The material coefficients were obtained by minimizing the root mean square error (RMSE) between the experimental and numerical data using a simplex algorithm:

$$
\min \boldsymbol{F}(\boldsymbol{X})=R M S E=\sqrt{\frac{\sum_{i=1}^{n}\left(x_{\exp , i}-x_{\text {num }, i}\right)^{2}}{n}},
$$

where $\boldsymbol{X}$ is the set of material coefficients (c.f. Equation (2)), $x_{\exp , i}$ are the displacements from the experimental data and $x_{n u m, i}$ the displacements from the numerical data for corresponding pressures.

The obtained numerical results are compared with the experimental data [5] in Figure 2 for three different age groups, with the corresponding material coefficients (c.f. Equation (2)) given in Table 3. It is clear from the RMSE, which is below $2 \%$ for all cases, that the material coefficients determined from the optimization process describes the cornea material with reasonable accuracy. The minimum and maximum values (indicated in Table 3 as blue and red, respectively) for each material coefficient will be used for the sampling range in the POD analysis.

Table 3. Optimized material coefficients obtained from the optimization process for the different age groups (maximum values are shown in red, and minimum in blue).

\begin{tabular}{ccccc}
\hline & $C_{10}(\mathrm{MPa})$ & $k_{1}(\mathrm{MPa})$ & $k_{2}$ & $\mathrm{RMSE}$ \\
\hline Age Group 1 Set 1 & 0.0125 & 0.0158 & 281.0 & $1.36 \%$ \\
Age Group 1 Set 2 & $\mathbf{0 . 0 1 2 7}$ & $\mathbf{0 . 0 1 2 4}$ & 299.0 & $1.46 \%$ \\
Age Group 2 Set 1 & 0.0102 & 0.0707 & 335.0 & $0.18 \%$ \\
Age Group 2 Set 2 & 0.0068 & 0.0494 & 385.0 & $0.20 \%$ \\
Age Group 2 Set 3 & 0.0067 & 0.0508 & 297.0 & $0.93 \%$ \\
Age Group 3 Set 1 & 0.0108 & $\mathbf{0 . 1 3 1 0}$ & 387.0 & $0.23 \%$ \\
Age Group 3 Set 2 & 0.0085 & 0.1270 & 398.0 & $0.18 \%$ \\
Age Group 3 Set 3 & $\mathbf{0 . 0 0 4 5}$ & 0.1080 & $\mathbf{4 0 7 . 0}$ & $0.24 \%$ \\
Age Group 3 Set 4 & 0.0055 & 0.0740 & $\mathbf{2 4 7 . 0}$ & $0.32 \%$ \\
\hline
\end{tabular}




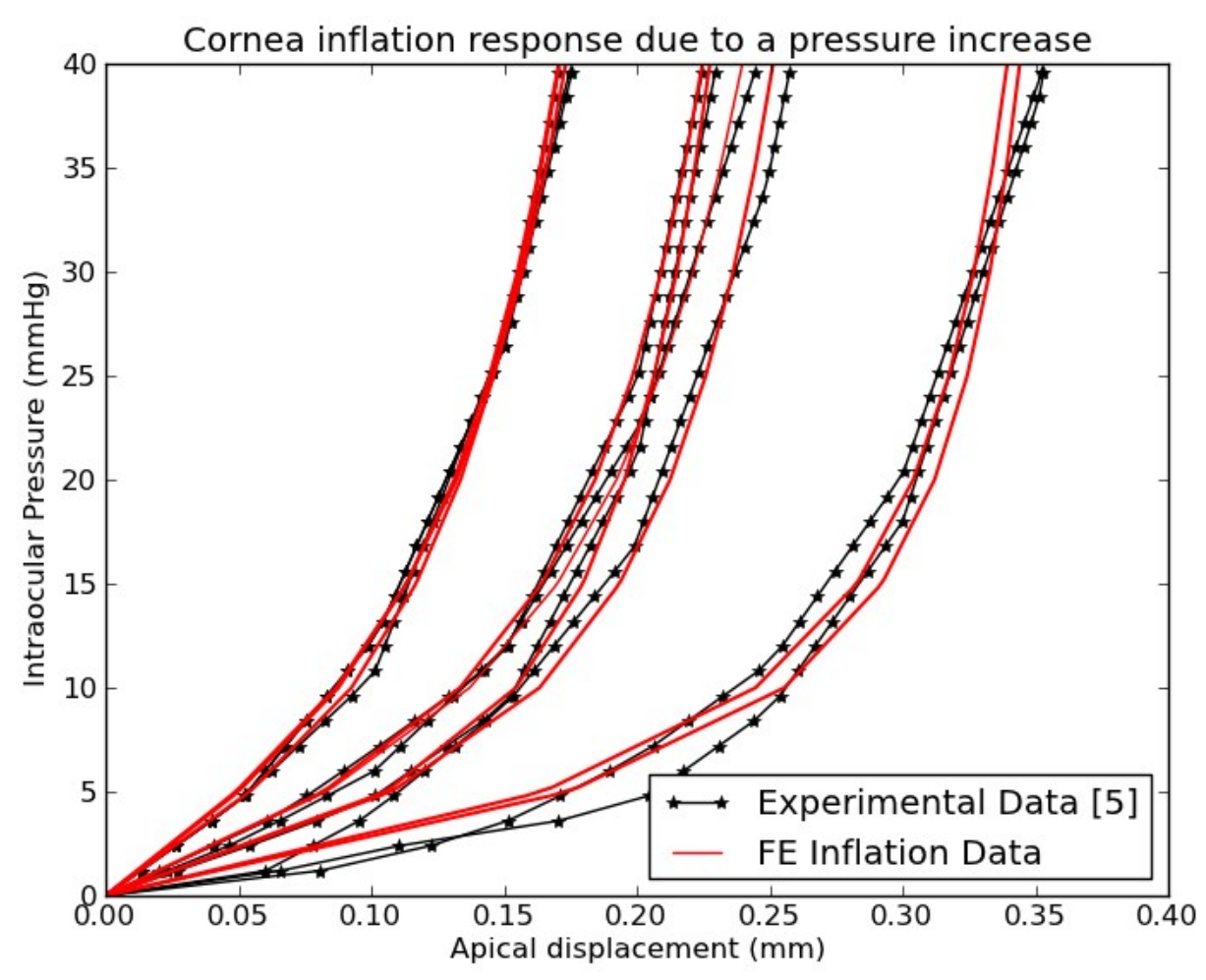

Figure 2: Calibration of optimized material coefficients from the FE inflation simulation with the experimental data [5].

\subsection{Application to Goldmann applanation tonometry}

Goldmann applanation tonometry is considered to be the 'gold standard' for determining intraocular pressure [8]. The principle of applanation is based on the Imbert-Fick principle which states that the pressure inside a spherical shell is equal to the force required to flatten a fixed area of the shell [7].

The applanation tonometry is simulated by means of a two step contact analysis (Figure 3(a) shows the Von Mises stress profile for each step). The intraocular pressure is applied to the posterior surface of the cornea in the first step. In the second step the cornea is applanated by an applanating prism, which is defined as a rigid body. The ocular response history obtained is shown in Figure 3(b) for an average cornea. The obtained ocular response history for each GAT simulation will be the target response for the POD algorithm.

From Figure 3(b) the intraocular pressure from the GAT simulation (IOPG) can be calculated from the Imbert-Fick principle:

$$
I O P G=\frac{\text { Applanation Force }}{\text { Applanated Area }}=\frac{0.0179 \mathrm{~N}}{7.35 \mathrm{~mm}^{2}}=\frac{0.002435 \mathrm{MPa}}{133.28 \mathrm{E}-06}=18.273 \mathrm{mmHg}
$$

By substituting the final applanating force value $(0.017926 \mathrm{~N})$ from Figure $3(\mathrm{~b})$ and an 
applanating area of $7.35 \mathrm{~mm}^{2}$, the IOPG is found to be $18.273 \mathrm{mmHg}$ (Age Group 2 Set 2 in Table 4). This method of calculating the IOPG was followed for the nine sets of optimized material coefficients from Table 3, using an average central corneal thickness (CCT) of $0.55 \mathrm{~mm}$ and applying an average intraocular pressure (IOP) of $16 \mathrm{mmHg}$. The results are shown in Table 4.

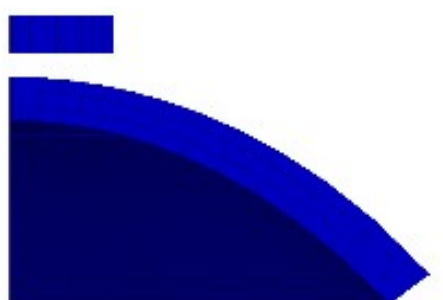

Initial state

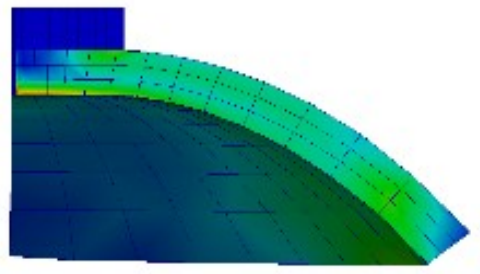

After applanation

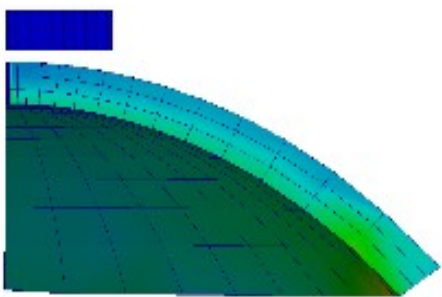

After IOP is applied

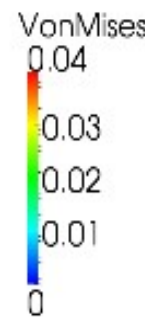

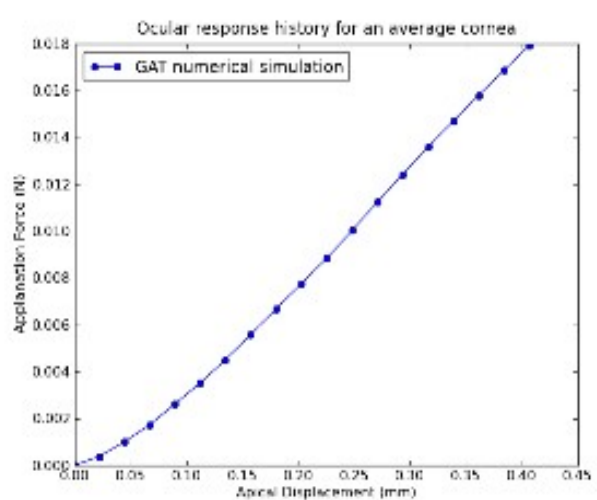

b) Ocular response history

a) Goldmann applanation tonometry numerical simulation

Figure 3: Numerical GAT simulation and ocular response history for an average cornea with $\mathrm{CCT}=0.55 \mathrm{~mm}$ and $\mathrm{IOP}=16 \mathrm{mmHg}$.

Table 4. Calculated IOPG for the nine different sets of optimized material coefficients.

\begin{tabular}{lcc}
\hline & IOPT $(\mathrm{mmHg})$ & $\begin{array}{c}\% \text { Error } \\
\text { (from IOPT }=16 \mathrm{mmHg})\end{array}$ \\
\hline Age Group 1 Set 1 & - & - \\
Age Group 1 Set 2 & 20.048 & $25.30 \%$ \\
Age Group 2 Set 1 & 18.968 & $18.55 \%$ \\
Age Group 2 Set 2 & 18.273 & $14.21 \%$ \\
Age Group 2 Set 3 & 18.197 & $13.73 \%$ \\
Age Group 3 Set 1 & 18.848 & $17.80 \%$ \\
Age Group 3 Set 2 & - & - \\
Age Group 3 Set 3 & - & - \\
Age Group 3 Set 4 & 17.430 & $8.94 \%$ \\
\hline
\end{tabular}

It is clear from Table 4, that the numerical GAT simulation does overestimate the IOPG, which is a known problem for this tonometry method [2, 7]. Also note that for three of the age group sets there are no reported values, this is because those material coefficient combinations were unable to obtain convergence with the associated intraocular pressure and central corneal thickness. 


\subsection{Proper orthogonal decomposition}

The previous study done by Ghaboussi et al. [6] approached the problem of determining the cornea material properties and the intraocular pressure as an inverse problem, where the ocular response history is known. This study builds on their work by utilizing a reduced order modelling technique, known as proper orthogonal decomposition (POD), instead of a combination of a genetic algorithm (GA) and artificial neural network (ANN) to estimate the intraocular pressure and material properties given an ocular response history.

POD is a mathematical procedure which aims to find lower order estimations for higher order systems. One of the most impressive features of the POD is that it uses a finite number of modes to capture the dominant components of a high dimensional system [10].

A POD uses a set of observations, or snapshots, which are then approximated by decomposing the system into a linear combination of basis functions:

$$
\boldsymbol{u}^{k} \approx \sum_{j=1}^{M} \alpha_{j}^{k} \psi_{j}(x),
$$

where $\alpha^{k}$ is a set of scalar correction coefficients relating to the $k^{\text {th }}$ snapshot, $\boldsymbol{u}^{k}$, and $\psi_{j}$ is the basis functions which is a function of the applanating displacement.

POD is used in this study to define a relationship between the cornea material properties, including the intraocular pressure, and the target response obtained from the GAT simulation. The entire procedure is shown schematically in Figure 4.

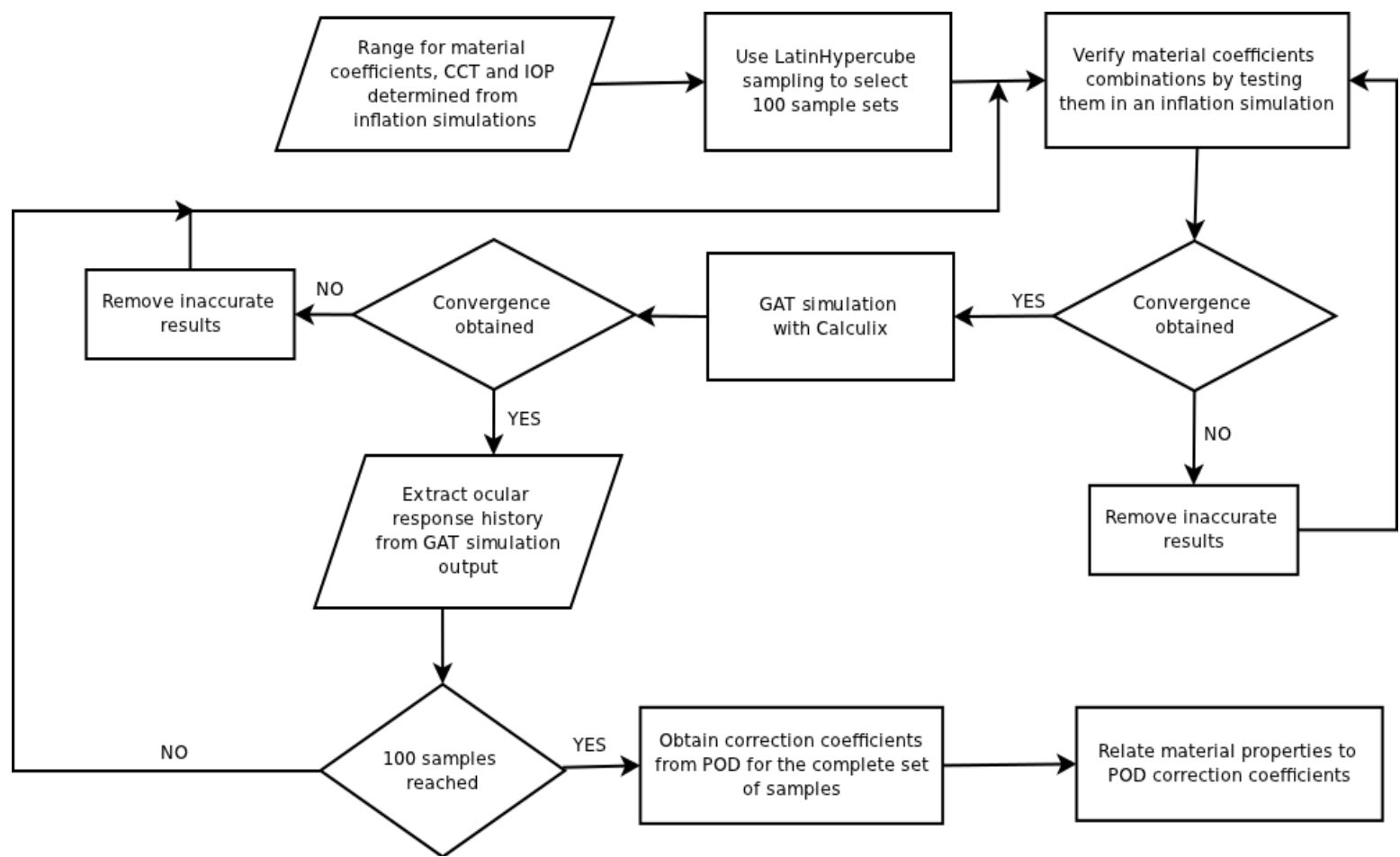

Figure 4: Schematic of the data generation and POD algorithm. 
Once this relationship is determined it is possible to estimate the material properties and intraocular pressure for an unknown ocular response.

The range for the three material coefficients, intraocular pressure and central corneal thickness (c.f. Table 3) that will be used as input to the GAT simulation, to obtain the various ocular response histories, is given in Table 5. The fixed values for the other material coefficients not being varied are also given. After verifying the material coefficient combinations that were sampled with Latin Hypercube sampling, 33 of the original 100 samples did not obtain convergence, and those material coefficient combinations were removed from the sample set. The POD algorithm was therefore only trained with 67 samples.

Finally to find the desired cornea material properties from a specific ocular response history, an inverse process is followed. The ocular response history is given as input to the POD algorithm. The relationship between material properties and the POD correction coefficients is then used to determine the corresponding cornea material properties and intraocular perssure of the ocular response history.

Table 5. Range of material coefficients, CCT and IOP used for GAT simulations.

\begin{tabular}{ccc}
\hline Properties & Range & Average \\
\hline C1 & $0.0045-0.0127 \mathrm{MPa}$ & $0.0086 \mathrm{MPa}$ \\
$\mathrm{D} 1$ & - & 0.004 \\
$\mathrm{k} 1$ & $0.0124-0.1310$ & 0.0717 \\
$\mathrm{k} 2$ & $247-407 \mathrm{MPa}$ & $327 \mathrm{MPa}$ \\
$\mathrm{CCT}$ & $0.45-0.65 \mathrm{~mm}$ & $0.55 \mathrm{~mm}$ \\
$\mathrm{IOP}$ & $10-24 \mathrm{mmHg}$ & $16 \mathrm{mmHg}$ \\
\hline
\end{tabular}

\section{RESULTS}

\subsection{POD performance}

The POD algorithm calculates the eigenvalues, which provide an indication of how much of the system information is captured by the associated modes. The number of modes that can be retained, while still providing a reasonable accuracy to describe the ocular response history, is found to be one. This is clearly illustrated in Figure 5, where it is seen that one mode (the largest eigenvalue) is sufficient independent of the number of samples used to 'train' the POD.

It is noted however that the accuracy of the POD estimations are dependent on the number of samples used to train it. The POD is able to more accurately predict the intraocular pressure if the material coefficient, intraocular pressure and central corneal thickness combinations, are chosen more widespread. 


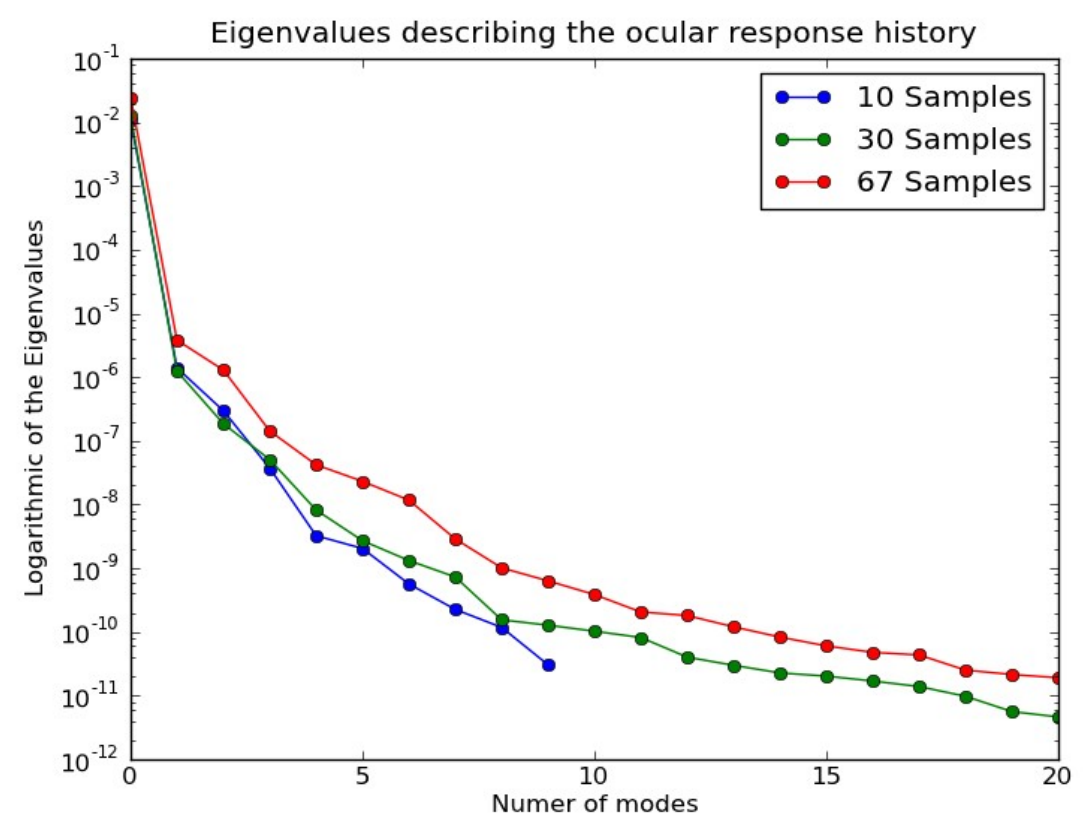

Figure 5: Eigenvalues describing the captured information from the ocular response history.

\subsection{Effect of material properties}

Several studies have shown that cornea material properties, such as central corneal thickness (CCT), do influence the intraocular pressure (IOP) measurement using Goldmann applanation tonometry $[2,7,9,12]$. A small study is conducted to determine whether the proposed POD technique does estimate the intraocular pressure while being insensitive to the central corneal thickness. To illustrate the effectiveness of this method, the results are also compared with those predicted using the ANN/GA combination [6], as well as the IOPG estimated from the numerical GAT simulation.

From Figure 6 we can conclude that the POD method is able to accurately predict the true IOP (i) for corneas with a CCT of $0.45 \mathrm{~mm}$, (ii) for corneas with a CCT of $0.55 \mathrm{~mm}$ and IOP of $16 \mathrm{mmHg}$ and $24 \mathrm{mmHg}$ and (iii) for corneas with a CCT of $0.65 \mathrm{~mm}$ and an IOP of $24 \mathrm{mmHg}$. It is clear for the cases of an IOP of $11 \mathrm{mmHg}$ and $16 \mathrm{mmHg}$ that the POD method overestimates the IOP with an increase in CCT, which is expected as it is a well known problem for GAT $[2,7]$. Finally for higher IOPs (IOP $=24 \mathrm{mmHg}$ ) the POD method performs rather well in comparison to the numerical GAT and the predicted IOP from the ANN/GA combination [6]. Unfortunately for lower IOPs (IOP $=11 \mathrm{mmHg}$ and $16 \mathrm{mmHg}$ ) the POD overestimates the true IOP with an increase in $\mathrm{CCT}$, and consequently performs poorly in comparison with the ANN/GA combination [6], but better than the numerical GAT simulation. It is clear however that the POD method is not entirely insensitive to central corneal thickness. 


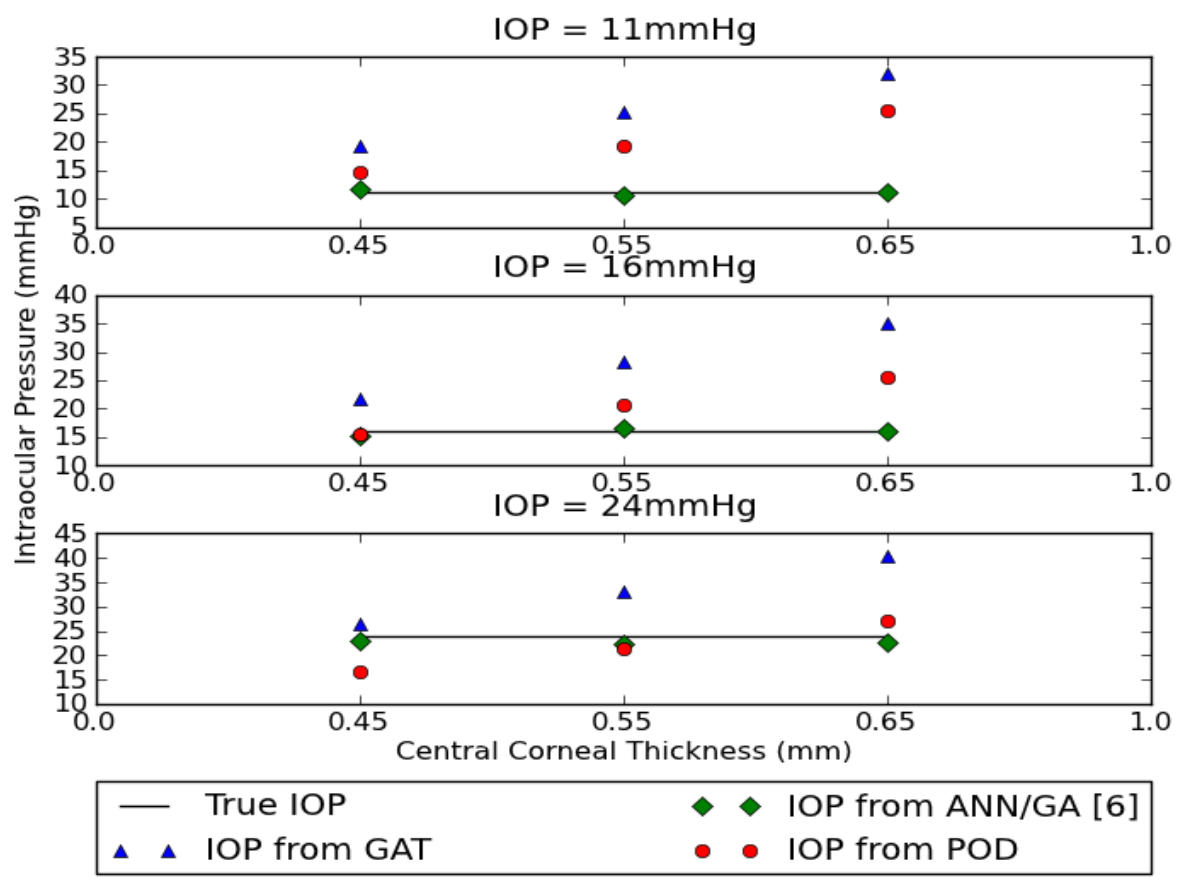

Figure 6: Effect of central corneal thickness for a true IOP of $11 \mathrm{mmHg}, 16 \mathrm{mmHg}$ and $24 \mathrm{mmHg}$.

\section{DISCUSSION}

Goldmann applanation tonometry is considered to be the 'gold standard' for measuring the intraocular pressure. Unfortunately this technique is known to be sensitive to cornea material properties, such as central corneal thickness. To address this problem, Ghaboussi et al. [6] proposed to use a combination of a genetic algorithm (GA) and an artificial neural network (ANN), which concluded that numerical techniques could be powerful tools to accurately estimate intraocular pressure with only a slight sensitivity to cornea material properties. However their proposed technique is not ideal as it is a stochastic routine, which can cause ambiguities. This work proposed to use a POD technique, which is more deterministic.

The results indicated that the POD technique does predict the true intraocular pressure rather accurately for thinner corneas, with sensitivities to the cornea material properties that deviate from the norm (IOP of $16 \mathrm{mmHg}$ and CCT of $0.55 \mathrm{~mm}$ ) considereably. When compared to the results obtained by Ghaboussi et al. [6], it can be concluded that this method is reasonably accurate, especially for thinner corneas and average to higher intraocular pressures. Even though the POD method does overestimate the intraocular pressure with an increase in central corneal thickness, it is also clear that it accurately predicts the true intraocular pressure for larger intraocular pressures (IOP $=24 \mathrm{mmHg}$ ).

This study showed that POD is a reliable method to use to predict the true intraocular 
pressure, as it does perform better than the numerical GAT simulation and shows promise when predicting higher intraocular pressures. The POD method is by no means perfect and still requires some improvements to increase the accuracy of its predictions. This study however did prove that POD can be used as a reliable technique to estimate the true intraocular pressure.

Future studies will focus on improving the accuracy of the predicted intraocular pressure from the POD method. This will be done by (i) improving the GAT numerical simulation, (ii) investigating different interpolation schemes to relate the material properties to the POD correction coefficients, and (iii) determine the optimum number of training samples.

\section{REFERENCES}

[1] Carney L.G., Mainstone J.C., Henderson B.A., "Corneal Topography and Myopia: A Cross-Sectional Study”. Investigative Ophthalmology \& Visual Science. 38, 311-320, 1997.

[2] De Moraes C.G.V., Prata T.S., Liebmann J., Ritch R., "Modalities of Tonometry and their Accuracy with Respect to Corneal Thickness and Irregularities". Journal of Optomology. 1, 43-49, 2008.

[3] Dhondt G.D.C., "Calculix: A Free Software three-dimensional structural finite element program". 1998.

[4] Elsheikh A., Wang D., Kotecha A., Brown M., Garway-Heath D., "Evaluation of Goldmann Applanation Tonometry using a nonlinear finite element ocular model". Annals of Biomedical Engineering. 34, 1628-1640, 2006.

[5] Elsheikh A., Wang D., Pye D., "Determination of the Modulus of Elasticity of the Human Cornea". Journal of Refractive Surgery. 23, 808-818, 2007.

[6] Ghaboussi J., Kwon T.H., Pecknold D.A., Hashash Y., “Accurate intraocular pressure prediction from applanation response data using genetic algorithm and neural networks". Journal of Biomechanics. 42, 2301-2306, 2009.

[7] Goldmann H., Schmidt T.H., "Über Applanationstonometrie”. (Ophthalmologica. 134, 221-242, 1957), in Classic Papers in Glaucoma, Ritch R., Caronia R.M. (eds.), Kugler Publications, The Hague, The Netherlands, 2000.

[8] Kniestedt C., Punjabi O., Lin S., Stamper R.L., "Tonometry through the ages". Survey of Ophthalmology. 53, 568-590, 2008.

[9] Kwon T.H., Ghaboussi J., Pecknold D.A., Hashash Y.M.A., "Effect of cornea material stiffness on measured intraocular pressure". Journal of Biomechanics. 41, 1707-1713, 2008.

[10] Liang Y.C., Lee H.P., Lim S.P., Lin W.Z., Lee K.H., Wu C.G., "Proper orthogonal 
decomposition and its application - Part 1: Theory". Journal of Sound and Vibration. $252,527-544,2002$.

[11] Liou H.L., Brennan N.A., "Anatomically accurate, finite model eye for optical modeling”. Journal of the Optical Society of America. 14, 1684-1695, 1997.

[12] Liu J., Roberts C.J., "Influence of corneal biomechanical properties on intraocular pressure measurement". Journal of Cataract and Refractive Surgery. 31, 146-155, 2005.

[14] Pandolfi A., Holzapfel G.A., "Three-Dimensional Modeling and Computational Analysis of the Human Cornea Considering Distributed Collagen Fibril Orientations". Journal of Biomechanical Engineering. 130, 061006-1 - 061006-12, 2008.

[15] Quigley H.A., Broman A.T., "The number of people with glaucoma worldwide in 2010 and 2020". British Journal of Ophthalmology. 90, 262-267, 2006.

[16] Rodrigues M.M., Warring III G.O., Hackett J., Donohoo P., "Cornea”. In Ocular Anatomy, Embryology and Teratology, Jakobiec F.A. (ed). Harper \& Row Publishers, Inc., Philadelphia, 1982. 